\title{
A Cellular Model for the Evolutionary Development of Lightweight Material with an Inner Structure
}

\author{
Till Steiner \\ Honda Research Institute \\ Europe $\mathrm{GmbH}$ \\ Carl-Legien-Str. 30 \\ 63073 Offenbach, Germany \\ till.steiner@honda-ri.de
}

\author{
Yaochu Jin \\ Honda Research Institute \\ Europe $\mathrm{GmbH}$ \\ Carl-Legien-Str. 30 \\ 63073 Offenbach, Germany \\ yaochu.jin@honda-ri.de
}

\author{
Bernhard Sendhoff \\ Honda Research Institute \\ Europe $\mathrm{GmbH}$ \\ Carl-Legien-Str. 30 \\ 63073 Offenbach, Germany \\ bs@honda-ri.de
}

\begin{abstract}
We present a model of simulated evolutionary development on the basis of cells as building blocks for growth. In this model, cells grow and interact in a three-dimensional (3D) environment, where development is controlled by a simple genome. Using cell-cell interaction such as differential adhesion, cells sort and form complex arrangements. This developmental process is evolved using a multi-objective evolutionary algorithm to achieve lightweight and stable material with a complex inner structure.
\end{abstract}

\section{Categories and Subject Descriptors}

I.6.3 [Computing Methodologies]: Simulation and Modeling-Applications

\section{General Terms}

Algorithms, Design

\section{INTRODUCTION}

Developmental models for cellular growth are expected to be a powerful alternative to direct encoding schemes for evolutionary computation. In developmental approaches, the genotype does not directly encode every detail of the final characteristics of individual solutions. Instead, the chromosomal information is used only to control the individual development, while the environment, such as simulated forces, pressure or temperature, or intercellular communication influence the final phenotypic appearance. These methods are generally termed simulated evolutionary development, or evolutionary embryogeny. It is believed that developmental approaches facilitate a more compact description length of complex systems and thus promote scalability [4]. In addition, developmental approaches inherently take environmental changes into account, which will increase both the robustness and the variability (developmental plascticity) of the evolved solutions.

Permission to make digital or hard copies of all or part of this work for personal or classroom use is granted without fee provided that copies are not made or distributed for profit or commercial advantage and that copies bear this notice and the full citation on the first page. To copy otherwise, to republish, to post on servers or to redistribute to lists, requires prior specific permission and/or a fee.

GECCO'08, July 12-16, 2008, Atlanta, Georgia, USA.

Copyright 2008 ACM 978-1-60558-130-9/08/07...\$5.00.
Among the various cellular models that have been proposed, some focus on the evolution of the control mechanism for the developmental process, see, e.g., [17], [3], [9]. In these models, the main target is to find an evolvable genetic representation that generates dynamics controlling cellular development. Therefore, the used cellular model is usually rather simple and abstract, where the cells are represented by spheres or other geometric shapes on a two or three dimensional grid [9], [13]. In some cases, the genetic control processes are studied from a purely theoretical point of view without using any multi-cellular model $[11,1]$.

On the other hand, other cellular models are more concerned with the development of multicellular structures. Fleischer [10] investigates a developmental model that includes differential cell adhesion and cell-cell signaling to produce desired shapes. Eggenberger [8] also describes a cellular model that is able to grow different kinds of shapes. An interesting application of cellular models has been presented by Bongard [2], who evolves 3D cellular arrangements for structuring robotic agents. Because of the necessity to simulate the physical cellular interactions that come with the growth process, these detailed cellular models often incur a high computational cost, which may impose a problem when they are embedded in an evolutionary framework, where these time-consuming calculations need to be performed for many individuals over generations. To relieve this problem, either a simpler cellular model has to be adopted, or massive parallel computational resources must be made available.

In this paper, we present a first step toward using evolutionary development for the design of complex structures. A developmental cellular model is evolved for optimizing the inner structure of lightweight and stable materials. The design of inner structures poses two main difficulties for conventional evolutionary algorithms. First, it is nontrivial to find an appropriate direct representation for describing the inner structure of a material. Second, the number of design parameters for describing the inner structures will increase dramatically both with the complexity of the structure and the task as well as with the size of the material. The latter aspect becomes particularly important if the structure should not be simply repetitive, e.g. like a honeycomb structure.

Different approaches have been reported for the optimization of the inner structure of cellular material $[16,15]$. These approaches usually pre-define the number of voids or number of combinable elements, thus making the optimization problem more tractable. A recent approach [14] uses a de- 
velopmental method to place a variable number of voids inside an optical fiber structure. However, the position of the holes (voids) is directly encoded in the genome, which implies that the genome length increases with the complexity of the represented structure.

We propose here a cellular model adapted from our previous work [17] for optimizing the inner structure of materials. The model consists of two cell types, one of which is a material cell with mass, and the other is a void cell. The development process starts with a few cells of both type positioned in the center of the calculation area. Cells divide recursively controlled by a regulatory gene that determines the type of the daughter cell. The regulatory gene has a number of receptors that sense the local information, including the type of the mother cell and the neighboring cells. After each cycle of cell division, the position of all cells will be re-calculated based on the physical interactions among the cells. In this way, a global internal structure will emerge through local cellular interactions. Since only very simple regulatory rules and a small number of parameters for cellular interaction are to be optimized, the dimension of evolutionary search is greatly reduced. Meanwhile, the encoding of the number of voids is no longer necessary, since this will be a natural result of cellular growth.

To evolve lightweight material structures using this model, two objectives need to be optimized. One is the structural stability, which is indicated by the maximal internal stress of the material when a certain force is applied to its surface. A finite element (FE) solver is employed to evaluate the internal tension of the evolved materials. The second objective is the mass of the materials, which is the number of the material cells. Note that the void cells do not have a mass and do not bear any force. Since these two objectives are conflicting with each other, we use NSGA-II [5], a popular evolutionary multi-objective algorithm to obtain a number of Pareto-optimal solutions. Our simulation results show that our cellular model is able to generate stable lightweight materials with complex inner structures.

The remainder of the paper is structured as follows. In Section II, we present the developmental cellular model that we use for our simulations. The concept and implementation for designing lightweight materials based on the cellular model are given in Section III. Section IV defines the experimental setup and presents the simulation results. A number of representative evolved structures are analyzed at the end of this section. We conclude the paper with a discussion of the results and an outlook in Section VI.

\section{SIMULATED CELLULAR DEVELOPMENT}

\subsection{Biological background}

Multicellular development starts with a fertilized egg cell. During the development, genes on the DNA control all cellular processes. Cells divide and use dynamic spatial information and cellular interaction to grow to their final differentiated state and contribute to the overall organism. Gene products do not only serve as building blocks for the cellular components, but also act as transcription factors (TFs) that are able to activate or repress the transcription of other genes. Therefore, the DNA encodes complex gene regulatory networks (GRNs) that govern all phases of development.
In a multicellular aggregation, cells interact with each other both chemically as well as physically. Chemical interaction takes the form of cell-cell signaling via diffusing TFs, which can then influence the expression of the genes in different cells. From a systems point of view, these diffusing TFs can be seen as local spatial and temporal coordinating signals for the control of the development of the organism. Although the coordination is mostly local, it finally determines the global organization of the cells.

Physical interaction of cells plays another crucial role during cellular development. It can take place passively, as well as actively. Passive physical interaction is a result of a cell being pushed toward another cell and thereby exerting a force on the latter. Active interaction takes place when a cell moves actively, or grows in its size. Another kind of active interaction that can be observed in biology is given by the production of cadherin molecules on the cells surfaces, which enables adhesion forces between cells to occur [12]. This adhesion is differential, i.e. cells with the same kind of surface molecules adhere more strongly to each other than to other cells. Differential adhesion leads to characteristic cellular distributions in multicellular aggregations, depending on the differential adhesion strengths. During biological development, this mechanism leads to the orderly separation of cell types and therefore facilitates the generation of organs. In general, differential adhesion can be seen as a complementary mechanism for the structuring of cellular distributions.

As mentioned in the introduction, the research efforts on simulating biological development in the evolutionary community are mainly motivated by two main potential benefits, namely, the ability to incorporate environmental knowledge, and the possibility to decrease the number of design parameters. For example, in our model, we use differential cell adhesion during the growth of multicellular structures, which facilitates the creation of complex inner structures with little encoded information. Although not yet implemented, it is straightforward to extend our model by including external information, such as positional information in terms of maternal TFs, or by using forces that a cell experiences as inputs to regulatory genes.

\subsection{Physical cell-cell interaction}

The cellular model used in this work grows in a 3D space. It is an extension from our previous model described in [17]. Since the simulation is computationally expensive, we use a domain decompositioning technique to achieve a speed-up, which makes it feasible to be included in an evolutionary framework. A few details about the model are presented in the following.

\section{- Passive interaction}

We model cells as spheres with a constant radius. The spheres impose a force on each other, depending on the distance between sphere centers, given by the nonlinear force function as described in the following equation:

$$
F=\left\{\begin{array}{ll}
d-2 r+0.022 & d \leq 2 r \\
\gamma_{*} \cdot 0.05 \cdot e^{\left(\frac{d-3 r}{0.5}\right)^{2}} & d>2 r
\end{array},\right.
$$

where $d$ is the distance between the cell centers, $r$ is the cell radius. $\gamma_{*}$ is a scaling factor used for differential cell adhesion, which is subject to evolution (find more details below). Note that spheres are allowed to 
overlap $(d \leq 2 r)$, in which case $F$ quickly reaches a negative sign and thus cells are pushed apart. A positive $F$ means adhesion of the cells. The constants in equation (1) are chosen experimentally so that they lead to a reliable growth process. Please note that this choice might influence the whole process, since the dynamic behavior of cells during cell sorting depends on the given equation. Sphere positions are constrained to a cube-shaped calculation area. All force vectors that act on a sphere are summed up, and the sphere positions are iteratively updated according to that resulting force. This leads to a minimal overlap positioning of the cells.

\section{- Active Interaction}

We use differential cell adhesion between different types of cells. Two different cell types, type A (void cell) and type B (material cell) are used in our simulations. Therefore, three parameters need to be evolved to affect cell adhesion: $\gamma_{A A}$ for scaling the adhesion force between two A-type cells, $\gamma_{B B}$ the adhesion force between two B-type cells, and $\gamma_{A B}$ the adhesion force between an A-type cell and a B-type cell. Scaling is achieved by replacing the factor $\gamma_{*}$ in equation (1) with the respective parameter for each case. Note that $0 \leq \gamma_{*} \leq 1$

\section{- Domain decomposition}

To minimize computational load, we subdivide the 3D space for development into 27 equally sized sub domains. These sub domains overlap at their boundaries. Since forces are only calculated between cells that belong to the same sub domain, the need for time consuming distance calculation and evaluation of equation (1) is heavily reduced. Thus, if a cell is pushed out of one domain into the next, it changes its neighborhood for force calculation. In this case, we choose the size of the overlap to be two times the cell radius, to reduce artifacts at domain boundaries. Please note that apart from these artifacts, this domain decompositioning has no effect on the growth process and the final cellular arrangement.

\section{- Cellular division}

Cellular division is simulated by placing a daughter cell close to the mother cell in a random direction, with a large overlap of both (the distance between centers is set to one fourth of the radius). Due to the big force those cells exert on each other, the daughter cell will be pushed apart from the mother cell after a few iterations.

\subsection{Genetic control of cellular development}

In [17], we present and analyze a model for the evolution of GRNs. Since the focus of this paper lies in the cellular model and its application, we use a much simplified version of the GRN, consisting of a virtual DNA (vDNA) with a single gene, which contains one regulatory unit and one structural unit. The regulatory unit has a number of receptors that sense the type of the mother cell and the type of the neighboring cells. The state of the regulatory unit is determined by four values in the genome: $w_{1}, w_{2}, \theta$, and $\sigma$, plus two inputs, i.e., mother cell $T^{1}$ and the mean value of surrounding cell types $T^{o}$ as follows:

$$
\text { eval }=\frac{\left(w_{1} \cdot T^{1}+w_{2} \cdot T^{o}\right)+2}{4},
$$

where $T^{1}$ can take the values -1 or 1 for A-type and B-type cells, respectively. $T^{o}$ is the sum of all $T$ values of cells adjacent to the mother cell divided by their number.

Depending on the regulatory state calculated from equation (2), the behavior of the structural unit can be described by the following pseudo-code, which determines the type of the daughter cell $\left(T^{d}\right)$ :

if $(\sigma>0.5)\{$

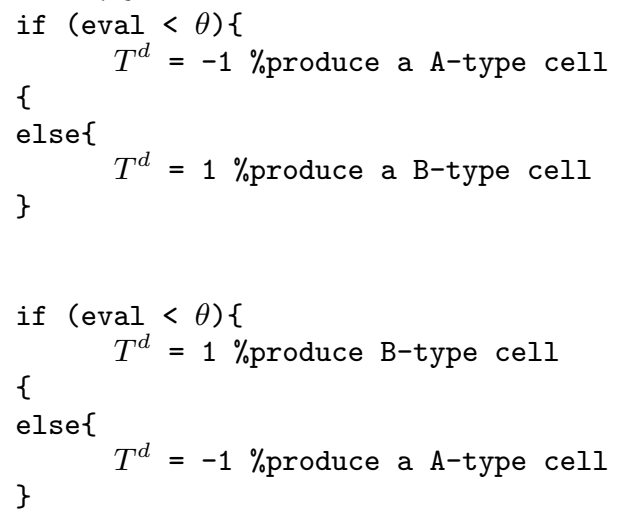

After the placement of all daughter cells inside the calculation area, the new positions of all cells are iteratively updated according to equation (1) to minimize overlap. Three parameters that specify the differential adhesion values, $\gamma_{A A}$, $\gamma_{B B}$ and $\gamma_{A B}$, are also encoded in the structural unit and are subject to evolution. Thus, in total there are seven parameters, $w_{1}, w_{2}, \theta, \sigma, \gamma_{A A}, \gamma_{B B}$ and $\gamma_{A B}$ to be evolved.

\subsection{The evolutionary algorithm}

In this work, NSGA-II [5] has been adopted for evolution, which is suited for optimizing multiple objectives in design. The reason behind this choice is that in our design, we intend to both minimize the weight of the designs and maximize their structural stability. NSGA-II is a popular evolutionary algorithm for solving multi-objective optimization problems. In our work, simulated binary crossover (SBX) [6] and polynomial mutation [7] have been employed to generate offspring. After the offspring population is generated, the elitist crowded non-dominated sorting is used for selecting parents for the next generation.

Different from single objective optimization algorithms, where often only one optimal solution is achieved, NSGA-II produces a set of Pareto-optimal designs, i.e. in our case, designs that trade their weight off against their structural stability. We will discuss in the next section how to evaluate the structural stability of such designs.

\section{DESIGN OF LIGHTWEIGHT STRUCTURES}

\subsection{Outline}

Two different cell types exist in our simulations. Cell type A (void cells) and cell type B (material cells). Through division, material cells and void cells grow until the whole 
calculation area is filled. Gene controlled cell growth, together with cell sorting due to cell-cell physical interaction, shapes inner structures. After the growth process has finished, the positions of the material cells represent nodes of a bar construction for mechanical stress calculation, while the void cells are removed since they represent holes in the structure. A force is then applied to the uppermost nodes of the bar construction and the stresses in the bars are calculated. The maximum stress indicates the structural stability of the material, which will be used as one of the objectives in the multi-objective optimization. The second objective is the weight (mass) of the structure, which is given by the number of material cells.

\subsection{From cell assembly to bar construction}

a)
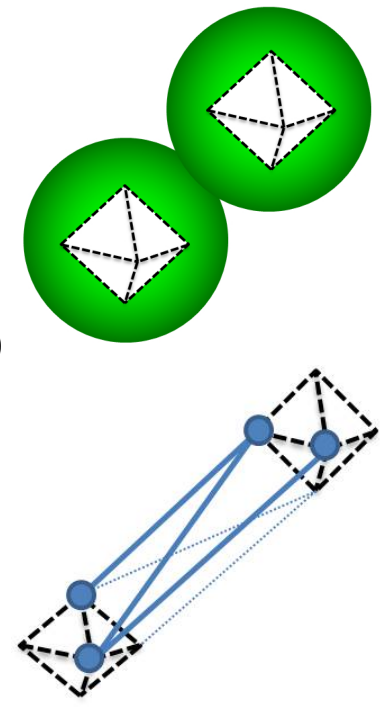

c)

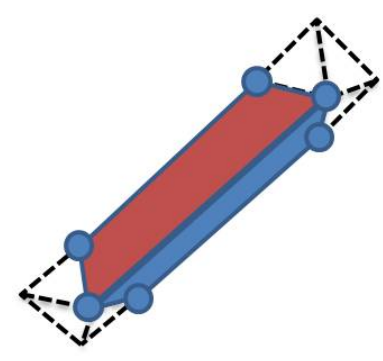

b)

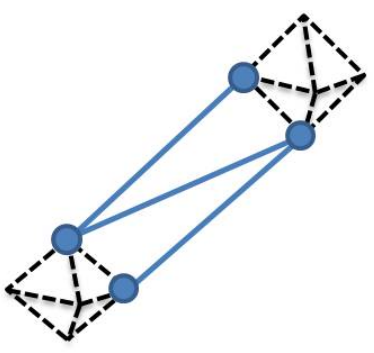

d)

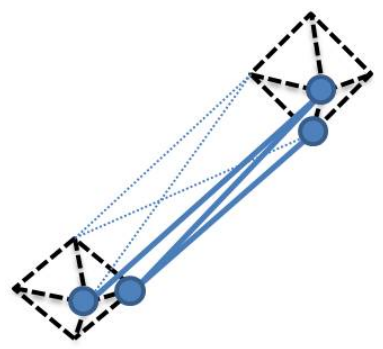

f)

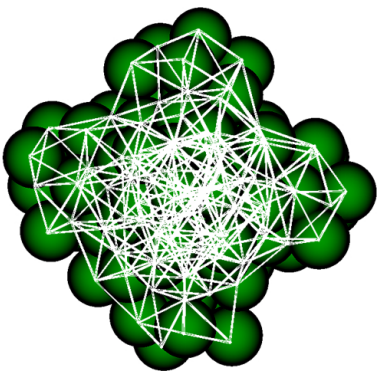

Figure 1: Diamond-shaped bar constructions replace cells for FE calculation. a) The diamonds are placed in the center of the cells. Sub-figures b) through e) show how consecutively three points of each of two diamonds are connected. Note that inner bars that make up the diamonds (dashed lines) are used for stress calculation only, their stresses are not considered for fitness calculation of the individual. Only connecting bars are used for that purpose. f) shows a sample cellular arrangement with the resulting bar construction.

To evaluate the stability of a cellular design, the arrangement of cells is converted into a structure that consists of bars, which are simple elements that can be used for finite element (FE) calculation. Fig. 1 illuminates how the cellular distribution is translated into the bar construction and how neighboring cells are connected. Cell centers are the centers of the diamonds that replace the cells for FE calculation. Two diamonds are connected if the centers have a distance less than or equal to two times the radius of the spheres. For the connection, three points of each diamond are chosen, according to the direction in which the neighboring cell lies. These points are then connected by a bar construction, which builds up a rigid connection between the diamonds. Fig. 1b) through e) shows how this is achieved in three consecutive steps. In each step, two points of each diamond are chosen and, if not connected previously, are connected by three bars (Fig. 1b). Therefore, a total of six bars will build up the connection. This is done for all neighboring cells.

A force $F$ is then distributed to the joints of the bar construction that are close to the upper boundary of the calculation area (distance less or equal two times the cell radius). Joints that are close to the lower boundary are fixed to their position. This implementation yields the same total force applied to all designs, independent of the actual number of cells that touch the upper calculation area boundary, i.e. cells to which the force is applied.
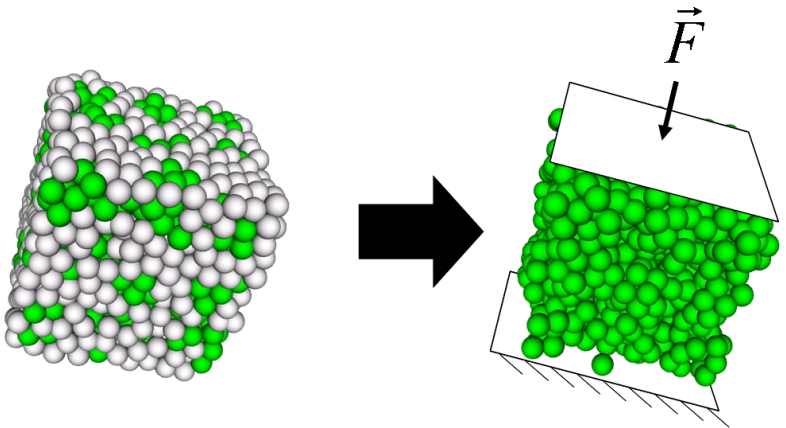

Figure 2: For stress calculation, the spacer cells are removed from the grown structure. Then, a force is applied on the design for evaluating the internal stresses using FE analysis.

The reason why we use this slightly complicated bar construction method is twofold. Firstly, the constructions built from any cellular arrangement can be used for FE calculations, because nodes are always properly connected and no ill-posed conditions can arise. Secondly, an automated translation from the cellular arrangement to the bar construction is conceivably easy because no exceptions for shape or meshing need to be considered. Therefore, the bar construction is a fast and reliable albeit not particularly elegant method to couple a growing cellular structure to a FE solver. For quality evaluation, we use the iterative solver for stress calculations included in the free Z88 FE-solver package ${ }^{1}$.

According to Fig. 1, two cells are connected by 6 bars. The complete stress between these cells is calculated by summing up the absolute value of all six stresses, neglecting the slightly different angle of the diagonal bars. This sum artificially penalizes solutions that contain torsional moments, which are characterized by opposing signs of the stresses in some of the 6 bars. Although the resulting sum is a good in-

\footnotetext{
${ }^{1}$ http://www.z88.org/
} 
dicator for stability, it renders the representation of the real stresses that would occur in the cellular arrangement impossible. Also note that stresses arising in the inner bars marked as dashed lines are, after stress calculation, not considered for fitness calculation. Only the bars that form connections between 2 different cells are taken into account. This reflects the conception of having solid cell centers possessing much higher stability than intercellular connections, so that a breaking of the structure would occur in between cells first.

\section{EXPERIMENTS AND RESULTS}

\subsection{Experimental setup}

For the experiment, we set the parameters for the FE solver as follows:

- Total force to be applied to upper cells: $10 \mathrm{~N}$

- Maximal number of FE solver iterations: 10000

- Residual error threshold to be reached: $\epsilon=1 \cdot 10^{-5}$

If a cellular arrangement is not suitable for calculation, e.g. there are not enough material cells to connect the upper and the lower borders, the solution is penalized by a high maximum stress value of $60 \mathrm{~N} / \mathrm{mm}^{2}$. The same maximum stress value is assigned to solutions for which the residual of the FE solver does not drop below a threshold, $\epsilon$, after 10000 iterations. The choice of $\epsilon$ is arbitrarily, but it leads to acceptable computation times and a sufficient number of converged solutions.

The population size for NSGA-II is set to 40 . The crossover probability is set to 0.5 and the distribution index for the SBX crossover is 20. Mutation probability is set to be inversely proportional to the chromosome length and the distribution index for mutation is also set to 20 . The simulation is run for 64 generations. To reduce computation time, fitness evaluations are parallelized on a cluster of 40 AMD Opteron computers with $2.4 \mathrm{GHz}$. The complete run takes little more than 4 hours. Cellular growth is initialized with two void cells and one material cell, placed in the center of the calculation area. After nine developmental cycles of cell division, a number of 1536 cells are produced that fill up the calculation area. The initial number of cells is determined arbitrary.

\subsection{Results}

The fitness values of individuals belonging to the first, the $30 \mathrm{th}$, and the last generation (64th) of the evolutionary run are depicted in Figure 3. Characteristic cellular distributions of four solutions are shown in Figure 5 and sample growth processes are depicted in Figure 7. These solutions exhibit qualitative differences, which can be traced along the Pareto front: The most stable solutions are built of massive blocks filled with B-type cells in the entire calculation area and thus has the largest weight. The most lightweight structures can be characterized as pole-shaped, where A-type cells fill most of the calculation area and thereby push B-type cells to one corner. Solutions in between show a complex inner structure, made up of blocks of B-type cells that are interspersed with A-type cells.

Massive, stable solutions and complex structured solutions are found early in the evolution (the first generation contains both these types of solutions), although the solutions with inner structures are above the Pareto front of the

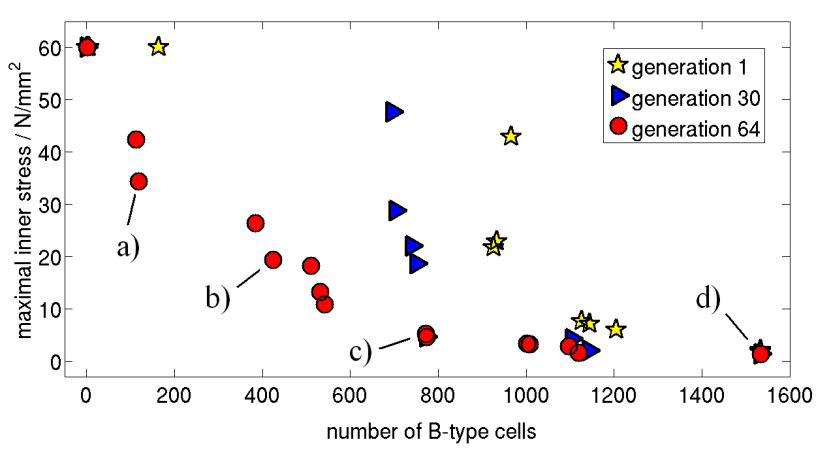

Figure 3: The fitness of the individuals belonging to the first, the $30 \mathrm{th}$, and the last generation. The two objectives - number of cells and maximal stress inside the structure - are minimized. The four solutions depicted in Figure 5a) through d) are marked by the respective character

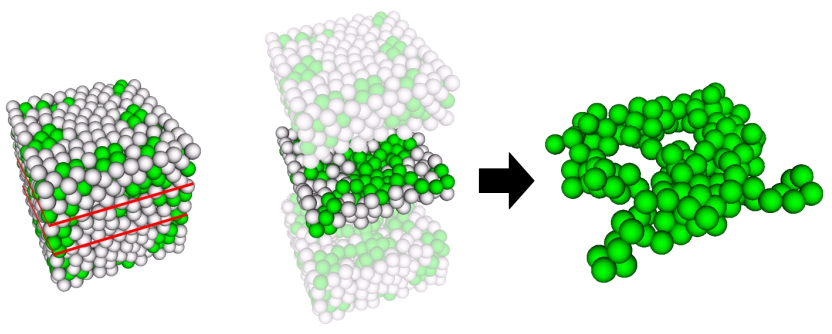

Figure 4: A section through the Pareto-optimal solution c) shown in Figure 5, reveals the complex inner structure. For better visibility, the viewing angle is changed and spacer cells are removed. Also, material cells are slightly magnified on the right hand side of the Figure.

final generation. The pole shaped solutions are found late during evolution (ca. generation 50).

\subsection{Analysis}

In the following, we analyze the genetic information that leads to the four sample solutions given in Fig 5. Table 1 gives the values of all variables in the genome of the four cases. The few parameters result in characteristically different structures. Before we look at these four cases, we will present some simple considerations that facilitate further analysis. For this purpose, please note that all values encoded in the genome are bounded to the range of $[0 . .1]$. Figure 6 shows three qualitatively different cell constellations. Each of these situations will cause a characteristic cell type of future daughter cells, depending on the values of the genetically encoded parameters. The first case characterizes a uniform cell A-type distribution (Figure 6a), the second case depicts a mixed cell types distribution (Figure 6b), and the third case delineates a uniform cell B-type distribution (Figure 6c). Table 2 gives a theoretical view of the possible resulting daughter cell types with consideration of different genetic values. The upper part of the table describes shapes that are governed by a $\sigma$ value larger than 0.5 . In this case, depending on the weight values $w_{1}$ and $w_{2}$ for the own cell 


\begin{tabular}{|c||ccccccc|}
\hline & $w_{1}$ & $w_{2}$ & $\theta$ & $\sigma$ & $\gamma_{A A}$ & $\gamma_{B B}$ & $\gamma_{A B}$ \\
\hline \hline a) & 0.1884 & 0.9910 & 0.4783 & 0.7442 & 0.9623 & 0.9679 & 0.3439 \\
b) & 0.1783 & 0.9844 & 0.4591 & 0.7442 & 0.9623 & 0.9679 & 0.2979 \\
c) & 0.9992 & 0.9912 & 0.5923 & 0.1670 & 0.0227 & 0.9415 & 0.0831 \\
d) & 0.1327 & 0.9482 & 0.8820 & 0.1541 & 0.8727 & 0.1265 & 0.3404 \\
\hline
\end{tabular}

Table 1: The values of the vDNA of the four selected individuals depicted in Figure 5

\begin{tabular}{|c|c|c|c|c|c|c|c|}
\hline \multicolumn{2}{|c|}{$w_{1}>>w_{2}$} & \multicolumn{6}{|c|}{$w_{1}<<w_{2}$} \\
\hline high $\theta$ & low $\theta$ & \multicolumn{3}{|c|}{ high $\theta$} & \multicolumn{3}{|c|}{ low $\theta$} \\
\hline case $1,2,3$ & case $1,2,3$ & case 1 & case 2 & case 3 & case 1 & case 2 & case 3 \\
\hline mother celltype & mother celltype & A cell & A cell & B cell & A cells & B cells & B cells \\
\hline \multicolumn{8}{|c|}{$\sigma \leq 0.5$} \\
\hline \multicolumn{2}{|c|}{$w_{1}>>w_{2}$} & \multicolumn{6}{|c|}{$w_{1}<<w_{2}$} \\
\hline high $\theta$ & low $\theta$ & \multicolumn{3}{|c|}{ high $\theta$} & \multicolumn{3}{|c|}{ low $\theta$} \\
\hline case $1,2,3$ & case $1,2,3$ & case 1 & case 2 & case 3 & case 1 & case 2 & case 3 \\
\hline opposite celltype & opposite celltype & B cell & B cell & A cell & B cells & A cells & A cells \\
\hline
\end{tabular}

Table 2: The different cases considered in the analysis of the three cases depicted in Figure 6.

a)

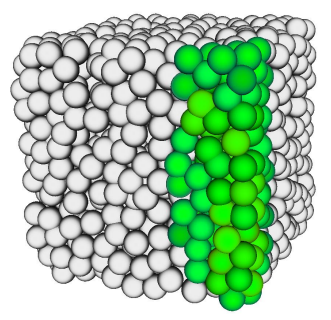

c)

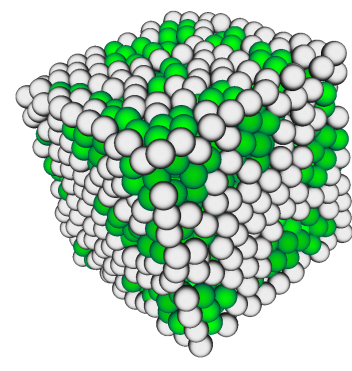

b)

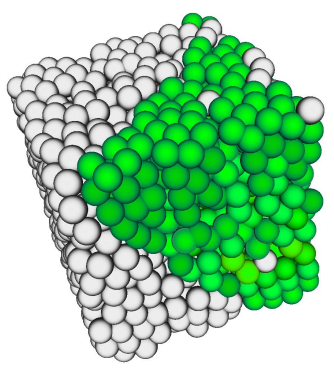

d)

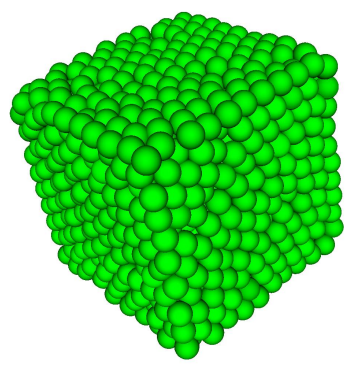

Figure 5: Different characteristic solutions can be traced along the Pareto-front. a) A very lightweight solution (118 B-type cells, $34.43 \mathrm{~N} / \mathrm{mm}^{2}$ ), b) a lightweight, more stable solution (424 B-type cells, $19.31 \mathrm{~N} / \mathrm{mm}^{2}$ ), c) stable, complex inner structure (773 B-type cells, $4.53 \mathrm{~N} / \mathrm{mm}^{2}$ ), and d) most stable and heaviest solution (1534 B-type cells, 1.29 $\mathbf{N} / \mathbf{m m}^{2}$ )

type and the surrounding cells types, different behaviors can be observed: If the influence of the own cell type dominates the influence of the surrounding cell types $\left(w_{1}>>w_{2}\right)$, each cell will simply produce daughter cells of the same type as its own. If the surrounding cell types influence is much higher than the own cell type $\left(w_{2}>>w_{1}\right)$, we need to distinguish the three cases presented in Figure 3 as follows:

In each case, the type of the daughter cells will be in-
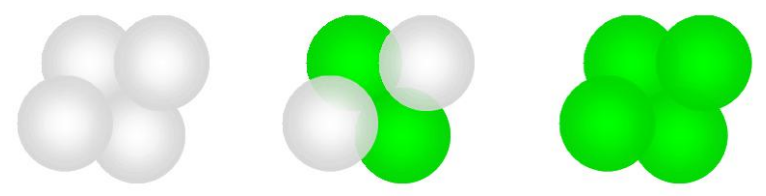

Figure 6: Three cases of cellular distributions that are considered for analysis. From left to right: Locally homogeneous A-type cellular distribution, inhomogeneous distribution, locally homogeneous BType cellular distribution

fluenced by the threshold value $\theta$ : if $\theta$ is high, case 1 and case 2 will lead to A-type cells, since eval in Equation (2) will be close to 0.25 for case 1 and close to 0.5 for case 2 , thus the high $\theta$ value will be larger than eval. Now imagine a situation where two equally sized homogeneous areas of the two different cell types are clearly separated and have a common border. The border would be 'pushed' toward the B side, since after division, the homogeneous A side, as well as both A- and B-type cells at the border would produce Atype cells. Only cells completely inside the homogeneous B side will produce B-type cells. Thus, after cellular division, relatively more A-type cells would be present than B-type cells.

If $\theta$ is low, the opposite effect takes place: the imaginary border between the areas will be pushed to the A side, since both types at the border will produce A cells. Thus, provided that in both cases, a strong differential A-A and B-B adhesion exists, cells will be caused to form larger or smaller compact blocks, and the whole calculation area will be separated into 2 homogeneous parts with sizes depending mainly on the value of $\theta$.

For a $\sigma \leq 0.5$ and $w_{1}>>w_{2}$ cells produce daughters with the opposite type compared to their own. In the cases where $w_{1}<<w_{2}$, the same thoughts as presented above yield the result that a high value of $\theta$ leads to a growth that breaks 
a)

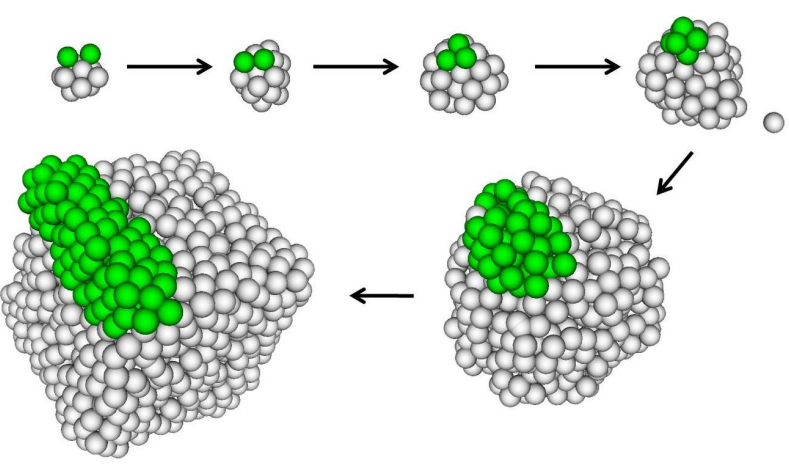

b)
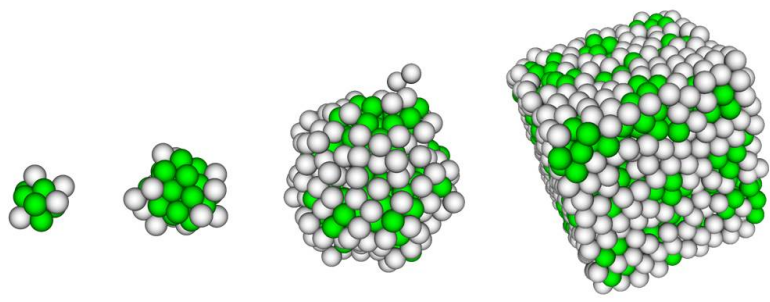

Figure 7: Sample growth processes of a) the lightweight solution (cf. Figure 5a) and b) a complex inner structure (compare Figure 5c). Note that individual distributions are chosen representatively and therefore not all nine developmental steps are shown.

even small clusters of A-type cells, mainly produces B-type daughter cells and only breaks very large clusters of B-type cells (cf. Table 2). A low value of $\theta$ will lead to the breaking of small clusters of B-type cells, where mainly A-type cells are produced and only large clusters of A-type cells are broken. Note that it is also possible that $\theta$ is less or greater than the total range of eval values. E.g., if $w_{1}=0$ and $w_{2}=0$, all $\theta>0.5$ are larger than eval. In such cases, all daughter cells produced during development will have the same type, independent of their own type and neighborhood, and just depending on the value of $\sigma$. On this basis, we will now investigate the solutions presented in Table 1 . The values of the first and the second solution resemble each other. This is also visible by an examination of the cellular distributions, refer to Figure 5a) and b). A similar growth process leads to the cellular distributions, with the difference that the final number of B-type cells in the second solution is a bit higher than in the first solution. $\sigma$ is greater than 0.5 . The differential adhesion values $\gamma_{A A}$ and $\gamma_{B B}$ explain the strict separation into the two domains. A $\theta$ smaller than 0.5 seems counterintuitive at first thought; a higher probability for Btype cells to be produced would result in a different cellular arrangement. An analysis of the growth process shows however, that an initial cell distribution of two A-type cells and one B-type cell results in eval lower than $\theta$ for all three cells $^{2}$. Therefore, all daughter cells are A-type cells. Physi-

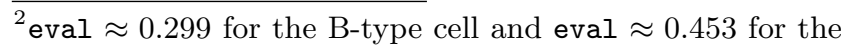

cal interaction after division pushes the B-type cell out of the adhering A cell structure. After two rounds of divisions, the force resulting from passive mechanical interaction pushes the B-type cell away from the A-type cell structure, since it is stronger than the adhesion between the two cell types. As a result, the B-type cell has no neighbors in the next when evaluating using Equation 2. Therefore, the resulting daughter cell is another B-type cell ${ }^{3}$. In this way, the two cell domains grow to their final distribution. This analysis shows that the initial distribution of cells is an important factor for the final cellular distribution.

The second structure in Figure 5 has a similar growth process, but because of the lower $\theta$ value, more B cells occur in the final cellular distribution.

According to Table 2, the third structure consists of Btype cells that adhere strongly, and A-type cells that adhere to B-type cells stronger than to themselves. This is a basis for a material structure that is interspersed with voids. $\sigma$ is less than 0.5 and $\theta$ slightly greater than 0.5 . This leads to a slightly higher probability of B-type cells to occur. Since $w_{1}$ and $w_{2}$ are both close to 1 , no differential weighting can be determined between own cell type and surrounding cell types for the calculation of the daughter cell type. The low $\sigma$ value and $\theta$ near 0.5 result in a break-up of medium size clusters both of A-type cells and B-type cells during division. Differential adhesion allows for the observed structure.

In the fourth structure, $\theta$ lies above the maximal value for eval in Equation $2\left(\right.$ eval $\left.^{\max } \approx 0.77\right)$. Because $\sigma<0.5$, the daughter cells in all possible cellular constellations will be B-type cells. Cellular adhesion does not play a big role in the overall cellular arrangement, since no cell sorting takes place. A similar result could be achieved by $\sigma>0.5$ and a very low $\theta$ value. In that case, the only importance would be that $w_{2}$ is much greater than $w_{1}$ and that $\|\theta\|_{2}>\left\|w_{1}+w_{2}\right\|_{2}$.

\section{SUMMARY AND CONCLUSION}

In this paper, we have applied a simplified version of an evolving gene regulatory network [17] for multi-cellular growth control to the domain of structure or topological optimization. The basic approach is to distinguish between two cell types - material cells and void cells - and to translate the cell assembly into a bar construction which can then be tested with standard FE simulations. Although the chosen task - minimization of the weight while maximising stress tolerance - is just one possible application for this method, it served us well to demonstrate the feasibility of this new approach to defining the inner structure of a design. We comprehensively analysed how different structures emerge from our model and through which parameter regimes the structure is determined. We believe this is important while it is frequently overlooked. We have to gain a better understanding how evolutionary development works in detail in order to properly assess its potentials as well as to develop the field further. We have to gain this understanding both for the emergent dynamics of the gene regulatory networks, see [17], as well as for the emergent multi-cellular formation.

Computational evolutionary development is still a young discipline, where both the modeling of cells as basic units of growth and the simulation of gene regulatory processes to control the growth are two main challenges for further re-

\footnotetext{
A-type cells

${ }^{3}$ eval $\approx 0.547$ for a single B-type cell.
} 
search. Several questions need to be addressed on the GRN level. For example, which are the dynamical behaviors that a GRN must exhibit to produce stable yet evolvable growth processes? What are the topographic properties of GRNs that lead to these dynamics? On the cellular modeling level, questions of a similar nature arise, e.g., which cellular models are useful for the evolution of certain structures? Which cellular models have better evolvability?

We believe that there is great potential in using evolutionary development for structural and topological optimization. However, it remains to demonstrate that the encouraging results presented in this work carry over to different kinds of realistic application domains.

\section{REFERENCES}

[1] U. Alon. An Introduction to Systems Biology - Design Principles of Biological Circuits. Chapman and Hall/CRC, 2006.

[2] J. Bongard and R. Pfeifer. Evolving complete agents using artificial ontogeny. Morpho-functional Machines: The New Species (Designing Embodied Intelligence), pages 237-258, 2003.

[3] C. Bowers. Formation of modules in a computational model of embryogeny. In Proceedings of the 2005 Congress on Evolutionary Computation (CEC'05), 2005.

[4] C. Bowers. Simulating Evolution with a Computational Model of Embryogeny (PhD Thesis). The University of Birmingham, 2006.

[5] K. Deb. Multi-Objective Optimization using Evolutionary Algorithms. Wiley, 2001.

[6] K. Deb and R. B. Agrawal. Simulated binary crossover for continuous search space. Complex Systems, 2(9):115-148, 1995.

[7] K. Deb and M. Goyal. A combined genetic adaptive search (geneas) for engineering design. Computer Science and Informatics, 4(26):30-45, 1996.
[8] P. Eggenberger. Evolving morphologies of simulated $3 \mathrm{~d}$ organisms based on differential gene expression. In Proceedings of the 4th European Conference on Artificial Life, 1997.

[9] D. Federici. Increasing evolvability for developmental programs. In J. Miller, editor, GECCO Workshop on Regeneration and Learning in Developmental Systems, 2004.

[10] K. Fleischer. A Multiple-Mechanism Developmental Model for Defining Self-Organizing Structures (PhD thesis). California Institute of Technology, 1995.

[11] N. Geard and J. Wiles. Investigating ontogenetic space with developmental cell lineages. In Proceedings of the Tenth International Conference on the Simulation and Synthesis if Living Systems, pages 56-62, 2006.

[12] S. Gilbert, editor. Developmental Biology. Sinauer Associates Inc., 2003.

[13] P. Haddow and J. Hoye. Achieving a simple developmental model for 3d shapes: Are chemicals necessary? In Proceedings of the GECCO'O', pages 1013-1020, 2007.

[14] S. Manos, L. Poladian, P. Bentley, and M. Large. A genetic algorithm with a variable-length genotype and embryogeny for microstructured optical fibre design. In Proceedings of the GECCO'06, pages 1721-1728, 2006.

[15] C. Mattheck. Design in der Natur - Der Baum als Lehrmeister. Rombach, 2006.

[16] C. Seepersad, J. Allen, D. McDowell, and F. Mistree. Multifuncitonal topology design of cellular material structures. ASME Journal of Mechanical Design, In Press, 2007.

[17] T. Steiner, L. Schramm, Y. Jin, and B. Sendhoff. Emergence of feedback in artificial gene regulatory networks. In Proceedings of the 2007 Congress on Evolutionary Computation (CEC'O'7), pages 867-874, 2007. 